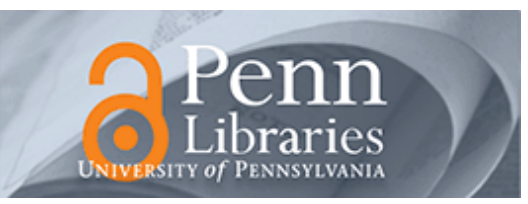

University of Pennsylvania

ScholarlyCommons

$4-16-2010$

\title{
Two-dimensional freezing criteria for crystallizing colloidal monolayers
}

\author{
Ziren Wang \\ Hong Kong University of Science and Technology \\ Ahmed M. Alsayed \\ University of Pennsylvania \\ Arjun G. Yodh \\ University of Pennsylvania \\ Yilong Han \\ Hong Kong University of Science and Technology
}

Follow this and additional works at: https://repository.upenn.edu/physics_papers

Part of the Physics Commons

\section{Recommended Citation}

Wang, Z., Alsayed, A. M., Yodh, A. G., \& Han, Y. (2010). Two-dimensional freezing criteria for crystallizing colloidal monolayers. Retrieved from https://repository.upenn.edu/physics_papers/32

\section{Suggested Citation:}

Wang, Z., A.M. Alsayed, A.G. Yodh and Y. Han. (2010). "Two-dimensional freezing criteria for crystallizing colloidal monolayers." The Journal of Chemical Physics. 132, 154501.

(c) 2010 American Institute of Physics. This article may be downloaded for personal use only. Any other use requires prior permission of the author and the American Institute of Physics.

The following article appeared in The Journal of Chemical Physics and may be found at http://dx.doi.org/10.1063/ 1.3372618 .

This paper is posted at ScholarlyCommons. https://repository.upenn.edu/physics_papers/32

For more information, please contact repository@pobox.upenn.edu. 


\title{
Two-dimensional freezing criteria for crystallizing colloidal monolayers
}

\author{
Abstract \\ Video microscopy was employed to explore crystallization of colloidal monolayers composed of \\ diameter-tunable microgel spheres. Two-dimensional (2D) colloidal liquids were frozen homogenously \\ into polycrystalline solids, and four 2D criteria for freezing were experimentally tested in thermal systems \\ for the first time: the Hansen-Verlet freezing rule, the Löwen-Palberg- Simon dynamical freezing \\ criterion, and two other rules based, respectively, on the split shoulder of the radial distribution function \\ and on the distribution of the shape factor of Voronoi polygons. Importantly, these freezing criteria, \\ usually applied in the context of single crystals, were demonstrated to apply to the formation of \\ polycrystalline solids. At the freezing point, we also observed a peak in the fluctuations of the \\ orientational order parameter and a percolation transition associated with caged particles. Speculation \\ about these percolated clusters of caged particles casts light on solidification mechanisms and dynamic \\ heterogeneity in freezing. \\ Disciplines \\ Physical Sciences and Mathematics | Physics

\section{Comments} \\ Suggested Citation: \\ Wang, Z., A.M. Alsayed, A.G. Yodh and Y. Han. (2010). "Two-dimensional freezing criteria for crystallizing \\ colloidal monolayers." The Journal of Chemical Physics. 132, 154501. \\ (C) 2010 American Institute of Physics. This article may be downloaded for personal use only. Any other \\ use requires prior permission of the author and the American Institute of Physics. \\ The following article appeared in The Journal of Chemical Physics and may be found at http://dx.doi.org/ \\ 10.1063/1.3372618.
}




\title{
Two-dimensional freezing criteria for crystallizing colloidal monolayers
}

\author{
Ziren Wang (王梓任), ${ }^{1}$ Ahmed M. Alsayed, ${ }^{2,3}$ Arjun G. Yodh, ${ }^{2}$ and Yilong Han (韩一龙) ${ }^{1, a)}$ \\ ${ }^{1}$ Department of Physics, Hong Kong University of Science and Technology, \\ Clear Water Bay, Hong Kong, China \\ ${ }^{2}$ Department of Physics and Astronomy, University of Pennsylvania, 209 South 33rd St., Philadelphia, \\ Pennsylvania 19104, USA \\ ${ }^{3}$ Complex Assemblies of Soft Matter, CNRS/UPENN/Rhodia UMI 3254, Bristol, Pennsylvania 19007, USA
}

(Received 29 December 2009; accepted 4 March 2010; published online 16 April 2010)

\begin{abstract}
Video microscopy was employed to explore crystallization of colloidal monolayers composed of diameter-tunable microgel spheres. Two-dimensional (2D) colloidal liquids were frozen homogenously into polycrystalline solids, and four 2D criteria for freezing were experimentally tested in thermal systems for the first time: the Hansen-Verlet freezing rule, the Löwen-PalbergSimon dynamical freezing criterion, and two other rules based, respectively, on the split shoulder of the radial distribution function and on the distribution of the shape factor of Voronoi polygons. Importantly, these freezing criteria, usually applied in the context of single crystals, were demonstrated to apply to the formation of polycrystalline solids. At the freezing point, we also observed a peak in the fluctuations of the orientational order parameter and a percolation transition associated with caged particles. Speculation about these percolated clusters of caged particles casts light on solidification mechanisms and dynamic heterogeneity in freezing. () 2010 American Institute of Physics. [doi:10.1063/1.3372618]
\end{abstract}

\section{INTRODUCTION}

Over the years, phenomenological freezing criteria derived from experiment and simulation have proved important for assignment of freezing points. ${ }^{1,2}$ One famous example is the Lindemann criterion $^{3}$ which has been widely used in three-dimensional (3D) melting and freezing, and its generalized version ${ }^{4}$ has been employed in studies of twodimensional (2D) melting. Simulations in 2D suggest four empirical criteria useful for identification of the freezing transition, none of which require free-energy calculations. They are, respectively, the 2D version of Hansen-Verlet (HV) freezing rule, ${ }^{5}$ the 2D dynamic Löwen-Palberg-Simon (LPS) criterion, ${ }^{6,7}$ the split second peak of the radial distribution function, ${ }^{8,9}$ and the bimodal distribution profile of the shape factor of Voronoi polygons. ${ }^{10}$ The $2 \mathrm{D}$ HV and LPS criteria have never been tested in experiment; however, they have been demonstrated in both equilibrium and nonequilibrium ${ }^{11}$ simulations with various particle interactions. The latter two criteria are not as well tested as HV and LPS; they have been verified in simulations of hard disks and, experimentally, in a qualitatively different nonthermal vibrating granular system. ${ }^{12}$

Besides empirical criteria, fundamental theories of freezing/melting in 2D have been formulated. KosterlitzThouless-Halperin-Nelson-Young (KTHNY) theory ${ }^{13-16}$ and other 2D melting theories, ${ }^{17-19}$ however, address singlecrystal melting. In principle, these melting theories can be applied to freezing, but in practice homogenous nucleation at finite quench rates most often produces polycrystalline solids upon freezing. Grain boundaries in polycrystalline solids

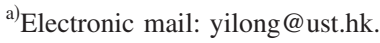

break both translational and orientational order, and the single-crystal melting criteria from KTHNY theory cannot be directly applied to this case. Indeed, it is interesting to note that the freezing transition from 2D liquid to 2D polycrystalline solids has hardly been studied by comparison to $2 \mathrm{D}$ single-crystal melting ${ }^{20}$ and 2D glass/jamming transitions. ${ }^{21-23}$

In this contribution we explore the freezing transition from 2D liquid to 2D polycrystalline solid, and we explore the utility of various empirical freezing criteria for prediction of freezing in this common thermal situation. In particular, we measure polycrystalline solidification in a colloidal monolayer, and we find that the four freezing criteria described above are useful to varying degrees for defining the freezing transition point. Moreover, we observe a peak in the fluctuations of the orientational order parameter at the freezing point, and a percolation transition of caged particles at the freezing point. These latter two observations led us to further speculations about freezing mechanisms in this colloidal system and could become useful empirical freezing criteria in their own right.

Colloids are outstanding model systems for melting and freezing studies, because the trajectories of individual particles in the samples are measurable by video microscopy. More often than not, however, colloids self-assemble to polycrystalline solids instead of monocrystals, especially if special annealing schemes are not employed. Thus an asymmetry exists between melting and freezing studies of 2D systems. In tests of theories of $2 \mathrm{D}$ single-crystal melting, ${ }^{20}$ experimenters typically anneal polycrystalline solids into very large crystalline domains using magnetic fields ${ }^{24}$ or shear. ${ }^{25}$ Subsequent observations are made only in the central areas of these large crystalline domains. ${ }^{24-27}$ Freezing experiments 


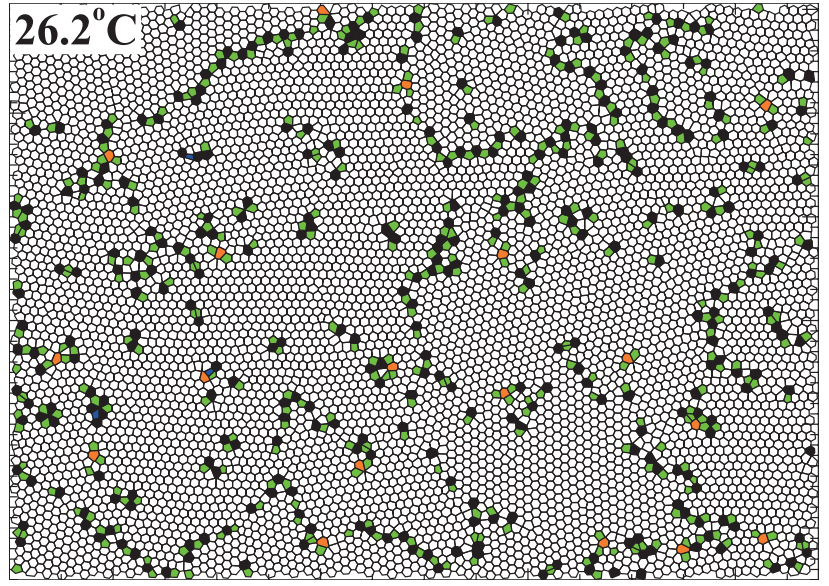

FIG. 1. A typical Voronoi diagram of the polycrystalline sample in $1 / 3$ of the full field-of-view at $26.2{ }^{\circ} \mathrm{C}$. Light green polygons: 5-neighboring particles; dark black: 7-neighboring particles; blue: 4-neighboring particles; orange: 8-neighboring particles.

are much more difficult than melting experiments, because polycrystalline solids cannot be annealed by external fields in advance nor can a central area of a crystalline domain be chosen a priori. Thus, most colloidal crystallization experiments involve polycrystalline solids and some nonequilibrium processes. Among these experiments are studies in 3D during recrystallization after shear melting ${ }^{28,29}$ and in a gravitational field, ${ }^{30}$ studies in $2 \mathrm{D}$ with electrohydrodynamic flows, ${ }^{31}$ studies with tunable depletion forces, ${ }^{32}$ and studies in rapidly quenched monolayers of magnetic spheres. ${ }^{33} 2 \mathrm{D}$ freezing has also been studied in dusty plasmas ${ }^{34,35}$ and driven granular systems. ${ }^{12}$

The thermally sensitive microgel spheres used in the present study generally freeze homogenously into polycrystalline solids. Their short-ranged particle interactions require long equilibration times to find global free-energy minima. The present work employs a high-resolution camera to study polycrystalline solidification over large areas $(\sim 23000$ particles) with typical polycrystalline solid domain sizes of about 1000 particles, see Fig. 1.

\section{MATERIALS AND METHODS}

Uniform ( $<3 \%$ polydispersity) rhodamine-labeled fluorescent $\mathrm{N}$-isopropyl acrylamide (NIPA) spheres were synthesized and suspended in $1 \mathrm{mM}$ acetic acid buffer solution. The resultant particles were sterically stabilized with short-ranged repulsive interactions. ${ }^{25}$ Their electrostatic repulsions were negligible in the buffer solution. Samples consisted of a monolayer of $\sim 0.8 \mu \mathrm{m}$ diameter NIPA microgel spheres confined between two glass walls separated at about $1 \mu \mathrm{m}$. The spheres floated around midplane of the cell because the density of sphere matches with the solution very well and because particle interactions with the sample cell walls are repulsive. Note, the gravitational height of the microgel particles, $h=k_{B} T /(\mathrm{mg})>100 \mu \mathrm{m}$, is much larger than the cell thickness. Here, $T$ is the room temperature, and $\mathrm{mg}$ is the buoyant weight of the microgel particle, which is quite small since water occupies more than $90 \%$ of the particle volume. The glass surfaces were rigorously cleaned so that particles did not stick to the walls. The samples were sealed and a fixed sample cell thickness was thereby frozen in. The wall separation varied by about $2 \mu \mathrm{m}$ over the $18 \times 18 \mathrm{~mm}^{2}$ sample area. Thus the walls can be considered to be parallel over the $\sim 0.1 \mathrm{~mm}$ field of view. The uniform monolayer nucleated homogenously, and flow was not detected during the experiment.

A 14-bit low-noise charge-coupled-device (CCD) camera was employed to record the motions of $\sim 23000$ spheres at $3.57 \mathrm{frame} / \mathrm{sec}$ in a $1392 \times 1040 \mathrm{pixel}^{2}$ (i.e., 147.3 $\times 110.2 \mu \mathrm{m}^{2}$ ) field of view. To obtain the best spatial resolution, we used confocal microscopy. Confocal microscopy avoided small image artifacts characteristic of bright-field microscopy and fluorescent bleaching problems endemic to traditional fluorescence microscopy. The temperature control (Bioptechs) on the microscope had $0.1{ }^{\circ} \mathrm{C}$ resolution. We decreased temperature from 29.5 to $24.1{ }^{\circ} \mathrm{C}$ in $0.3{ }^{\circ} \mathrm{C} /$ step and recorded $20 \mathrm{~min}$ video at each temperature after several minutes of equilibration. One-minute and 1-h equilibration times appeared to produce little difference. The particle positions in each frame were identified using standard image analysis algorithms. ${ }^{36}$

Diameters of soft spheres cannot be defined unambiguously. In order to estimate sphere packing fraction, we measure the particle diameter from the direct image data and calibrate at the close-packing area fraction. The diameter determined from the image analysis varied linearly with the temperature, consistent with the hydrodynamic particle diameter measured by light scattering. Note, however, the hydrodynamic diameter is obviously an overestimate of the true particle diameter, and the diameter from image analysis need not be perfectly accurate either. Hence we calibrated the sphere diameter at the close-packing state, defined as the position of the measured first peak of the radial distribution function $g(r)$ at the close-packing point. Figures 2(a)-2(c) show that the close-packing state at $26.2{ }^{\circ} \mathrm{C}$ has the best crystalline structure, i.e., it is characterized by the highest peak in the structure factor $S(k)$, minimum defect density, and maximum mean local orientational order parameter. The local 2D orientational order parameter ${ }^{20}$ of particle $j$ is

$$
\psi_{6 j}=\frac{1}{n n} \sum_{k=1}^{n n} e^{6 i \theta_{j k}}
$$

where $\theta_{j k}$ is the angle of the bond between particle $j$ and its neighbor $k . n n$ is the number of nearest neighbors identified from the Delaunay triangulation. Below $26.2{ }^{\circ} \mathrm{C},\left\langle\left|\psi_{6 j}\right|\right\rangle$ and $S(k)$ decrease slightly and the defect density increases slightly as shown in Figs. 2(a)-2(c), due in part to small out-of-plane buckling and soft-sphere lattice deformation. Combining the slope of the diameter-versus-temperature curve from image analysis and the calibrated diameter derived from $g(r)$ at $26.2{ }^{\circ} \mathrm{C}$ in the close-packing state, we find that the effective diameter $\sigma$ varies linearly to good approximation from $0.71 \mu \mathrm{m}$ at $29.5{ }^{\circ} \mathrm{C}$ to $0.92 \mu \mathrm{m}$ at $24.1^{\circ} \mathrm{C}$. The resultant particle area-fraction-versus-temperature curve is shown in Fig. 2(d). Here we use the more popular definition of the area fraction, $\rho=n \sigma^{2}$, without the factor $\pi / 4$. $n$ is the number density. 

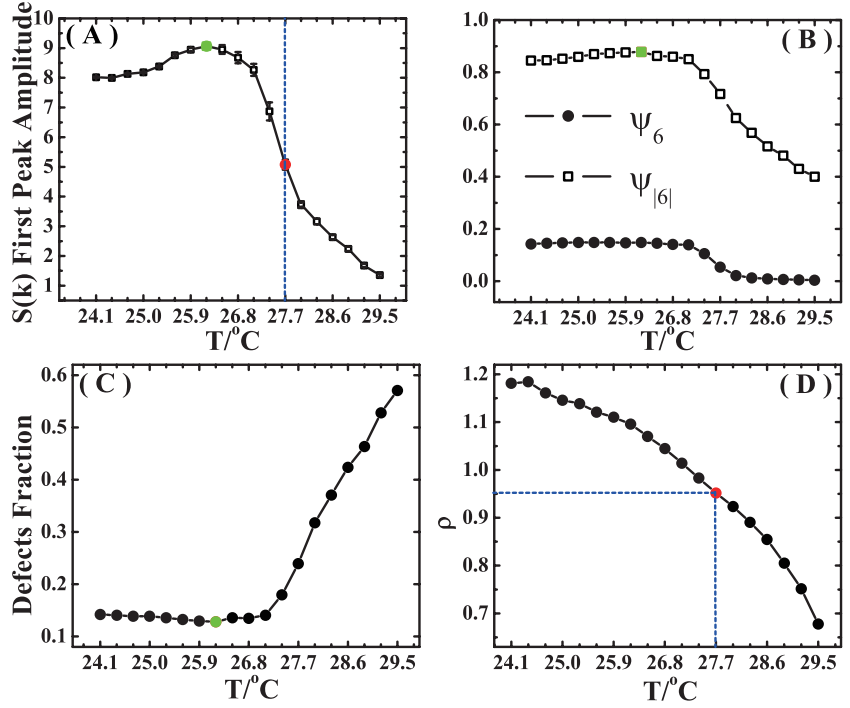

FIG. 2. (a) The height of the first peak of the structure factor $S(k)$. According to the $\mathrm{HV}$ freezing rule, the freezing transition is at $27.7^{\circ} \mathrm{C}$. The close packed state with best crystalline structure is at $26.2^{\circ} \mathrm{C}$. (b) Solid circles: the mean global orientational order parameters $\psi_{6}$; Open squares: the mean local orientational order parameter $\psi_{|6|}$ (see Sec. III E). (c) Defect fractions as a function of temperature. Defects are defined as particles without six nearest neighbors. (d) Area fractions $\rho=n \sigma^{2}$ as a function of temperature.

In the polycrystalline freezing, grain boundaries break up quasilong-ranged orientational order and, therefore, unambiguous identification of a hexatic phase is not possible. Thus, the freezing transition we have measured in our polycrystalline samples appears to be a first-order process, without hexatic phase. A similar behavior has been observed in the freezing of polycrystalline monolayers of magnetic spheres with dipolar interactions, ${ }^{33}$ wherein freezing appeared to be a first-order process evolving from solid to a coexistence phase at the melting point, and from liquid to a coexistence phase at the freezing point. In our samples, the coexistence phase was characterized by crystalline patches coexisting with fluid, similar to the coexistence phase observed in the monolayers of magnetic spheres. ${ }^{33}$ We note, however, that such patches do not unambiguously imply a first-order transition, because patches can also arise in dense fluid phases. ${ }^{2,25,37}$ Nevertheless, our measurements lead us to assign melting and freezing points at 26.8 and $27.7^{\circ} \mathrm{C}$, respectively. The difference in temperature suggests that a solid-liquid coexistence regime exists, and that the "transition" process is first-order. Freezing points are derived and discussed in Sec. III; they are the main subject of this contribution.

The melting point can be derived from the dynamic Lindemann parameter, ${ }^{4} L$, defined as

$$
L^{2}=\frac{\left\langle\left(\Delta \mathbf{r}_{\mathrm{rel}}(t)\right)^{2}\right\rangle}{2 a^{2}}=\frac{\left\langle\left(\Delta \mathbf{u}_{i}(t)-\Delta \mathbf{u}_{j}(t)\right)^{2}\right\rangle}{2 a^{2}},
$$

where $\Delta \mathbf{r}_{\text {rel }}$ is the relative nearest neighbor-neighbor displacement; $\Delta \mathbf{u}_{i}$ is the displacement of particle $i$, and particles $i$ and $j$ are nearest neighbors. This definition represents a slightly modified version of the traditional Lindemann parameter, because the traditional Lindemman parameter diverges in $2 \mathrm{D}$ due to strong long-wavelength fluctuations.

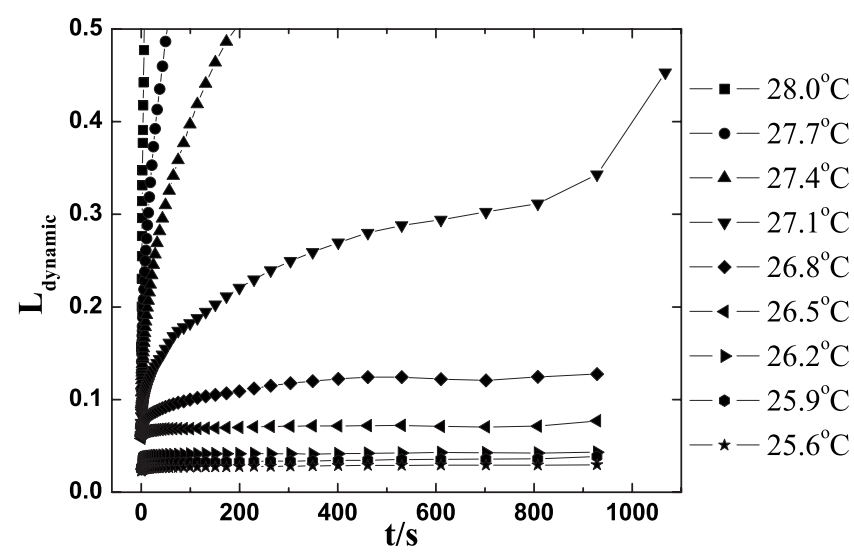

FIG. 3. The time-dependent dynamic Lindemann parameters plotted as a function of temperature. This criterion can be used to estimate the sample melting point. (Note, irrespective of its last time-point, the Lindemann parameter at $27.1{ }^{\circ} \mathrm{C}$ is too high for the system to be considered a solid.)

Figure 3 shows that the melting point falls within the temperature (and corresponding area fraction) range of $26.8-27.1{ }^{\circ} \mathrm{C}$ wherein $L$ is becoming divergent. At $26.8{ }^{\circ} \mathrm{C}$, $L$ saturates at $13 \%$, close to the simulation result of $12 \%$ for 2D dipole and 2D Lennard-Jones crystals. ${ }^{4}$

\section{RESULTS AND DISCUSSION}

\section{A. HV 2D freezing criterion}

To identify the freezing point, we first apply the 2D HV criterion, which is demonstrably robust in both equilibrium and weak nonequilibrium simulations. ${ }^{11}$ The $2 \mathrm{D}$ version of the $\mathrm{HV}$ freezing rule ${ }^{5}$ states that a 2D liquid freezes when the amplitude of the first peak of the structure factor $S(k)$ exceeds a critical value of approximately 5 . For different particle interactions, this critical value varies from 4.4 to 5.5. ${ }^{5,8,38}$ In $3 \mathrm{D}$, the critical values of the $\mathrm{HV}$ criterion is 2.85 . $^{39}$ Based on this freezing criterion, Fig. 2(a) shows a freezing point of $27.7 \pm 0.1{ }^{\circ} \mathrm{C}$. Notice, at this temperature, the slope of the curve is steepest. Such "steepest slopes" have also been observed in simulations at the freezing points of systems with Lennard-Jones and $r^{-12}$ potentials. ${ }^{5,8}$ At $27.7^{\circ} \mathrm{C}$, the area fraction $\rho=0.95$, is comparable to 0.89 for hard disks and 0.986 for $r^{-12}$-potential particles ${ }^{5}$ at the freezing point.

Figure 4 shows that the second peak of $S(k)$ starts splitting at the freezing point of $27.7^{\circ} \mathrm{C}$. Such a split has been observed in simulations of the freezing of repulsive $r^{-12}$ and $r^{-6}$ particles, ${ }^{5,8}$ but not in hard disks and Yukawa particles. ${ }^{38,40}$ Hence this latter feature is not considered a general freezing criterion.
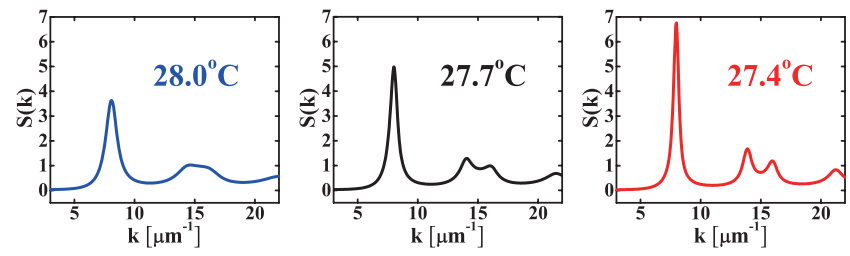

FIG. 4. Structure factors, $S(k)$, near the freezing point. At the freezing point, $27.7^{\circ} \mathrm{C}$, the first peak height reaches its critical value of 5 and satisfies the HV freezing rule, and the second peak develops a split peak structure. 


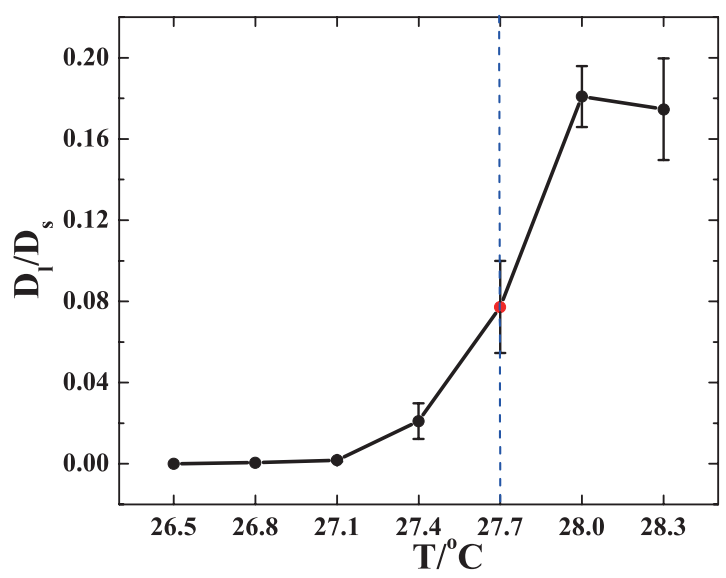

FIG. 5. The ratio of the averaged long-time diffusion coefficient to the short-time diffusion coefficient $D_{l} / D_{s}$. According to the LPS freezing criterion, the freezing point is at $27.7^{\circ} \mathrm{C}$ where $D_{l} / D_{s} \simeq 0.08$.

\section{B. LPS 2D dynamical freezing criterion}

The second freezing criterion is the dynamic LPS criterion for Brownian particles: ${ }^{6,7}$ a liquid freezes when the ratio of the averaged long-time diffusion coefficient to the averaged short-time diffusion coefficient, $D_{l} / D_{s}$, is larger than a critical value ranging between 0.07 and 0.1 in $2 \mathrm{D}$, depending on particle interaction. ${ }^{7,41}$ This criterion is fairly well established by simulation in both equilibrium and weak nonequilibrium systems. ${ }^{11}$ In 3D, the critical value of $D_{l} / D_{s}$ is $0.1,{ }^{6}$ a number that has been experimentally tested in the freezing of a 16-layer supercooled colloidal fluid. ${ }^{28}$

Here, we test the LPS criterion in 2D. Figure 5 shows that the empirically derived freezing point is also at $27.7 \pm 0.1^{\circ} \mathrm{C}$. Although our $D_{l}$ and $D_{s}$ have large error bars (because of limited short-time resolution and finite total observation time), the freezing point can still be accurately ascertained due to the steep slope at $D_{l} / D_{s} \simeq 0.08$.

\section{Second peak shoulder of the radial distribution function}

The third and the fourth (see Sec. III D) freezing criteria are less well tested than the first two criteria. They have mainly been studied in hard-disk simulations. The third criterion states that the second peak of the radial distribution function $g(r)$ should start to exhibit a shoulder within about $5 \%$ of the freezing point. ${ }^{8,9}$ The shoulder corresponds to the formation of hexagonally close-packed four-particle arrangements. ${ }^{9}$ The radial distribution function is defined as

$$
g(r=|\mathbf{r}|)=\frac{1}{n^{2}}\left\langle\phi\left(\mathbf{r}^{\prime}+\mathbf{r}, t\right) \phi\left(\mathbf{r}^{\prime}, t\right)\right\rangle,
$$

where $\phi=\sum_{j=1}^{N(t)} \delta\left(\mathbf{r}-\mathbf{r}_{j}(t)\right)$ is the distribution of $N$ particles in the field of view with area $A, n=\langle\phi\rangle=\langle N\rangle / A$ is the average particle number density. The angle bracket denotes an average over time and space. Figure 6 shows that the shoulder in $g(r)$ emerges at about $28.0{ }^{\circ} \mathrm{C}$, instead of the freezing point of $27.7^{\circ} \mathrm{C}$ predicted by most of the other criteria. The area fractions of 28.0 and $27.7{ }^{\circ} \mathrm{C}$, however, are different by only $3 \%$, see Fig. 2(d). Hence the third criterion roughly holds for the polycrystalline freezing of soft microgel spheres. This
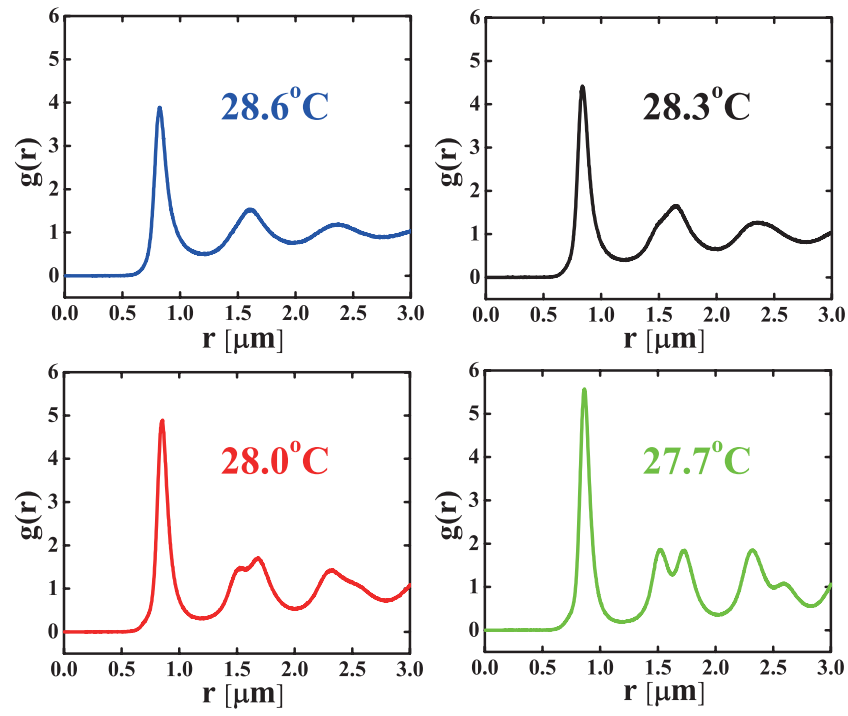

FIG. 6. The radial distribution function $g(r)$ near the freezing point. According to the third freezing criterion, the freezing area fraction is around $28.0^{\circ} \mathrm{C}$, where the second peak of $g(r)$ begins to split.

criterion has been numerically tested in hard disks $^{9}$ and Lennard-Jones systems ${ }^{8}$ with about $5 \%$ accuracy.

\section{Bimodal shape factor probability distribution}

The fourth criterion states that the probability distribution of the shape factors, $\xi$, of Voronoi polygons in the sample becomes bimodal near the freezing point. ${ }^{10}$ The shape factor is defined as

$$
\xi_{i}=C_{i}^{2} /\left(4 \pi S_{i}\right),
$$

where $S_{i}$ is the area and $C_{i}$ the perimeter of the Voronoi polygon of the $i$ th particle. ${ }^{10}$ Note, $\xi_{i}=1$ for circles, and $\xi_{i}$ $>1$ for all other shapes. The peak at small $\xi$ corresponds to the presence of regular hexagons in the sample, whereas the peak at large $\xi$ corresponds to pentagons and distorted hexagons in the sample. ${ }^{10}$

This criterion has been confirmed to be useful in harddisk simulations ${ }^{10}$ and in a vibrating nonthermal granular hard-disk experiment. ${ }^{12}$ Figure 7 shows that the bimodal

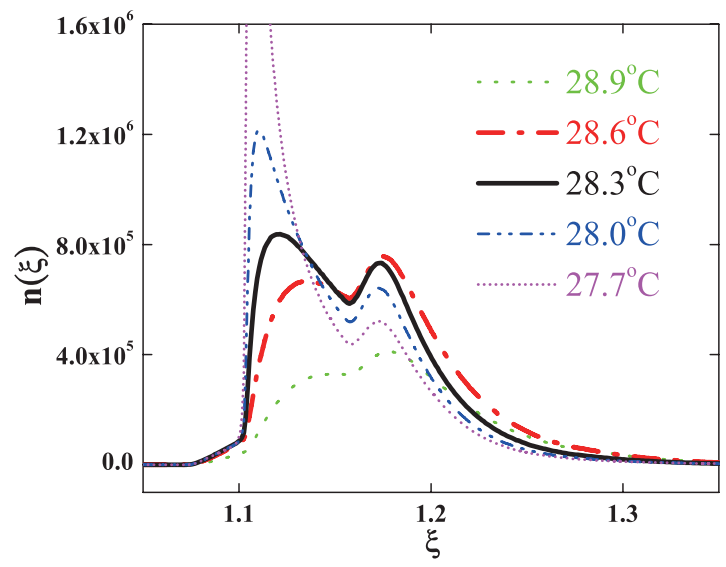

FIG. 7. The distributions of the shape factors $\xi$ of Voronoi polygons. According to the fourth freezing criterion, the bimodal distribution at $28.3{ }^{\circ} \mathrm{C}$ should be (and is) within 5\% of the freezing point. 


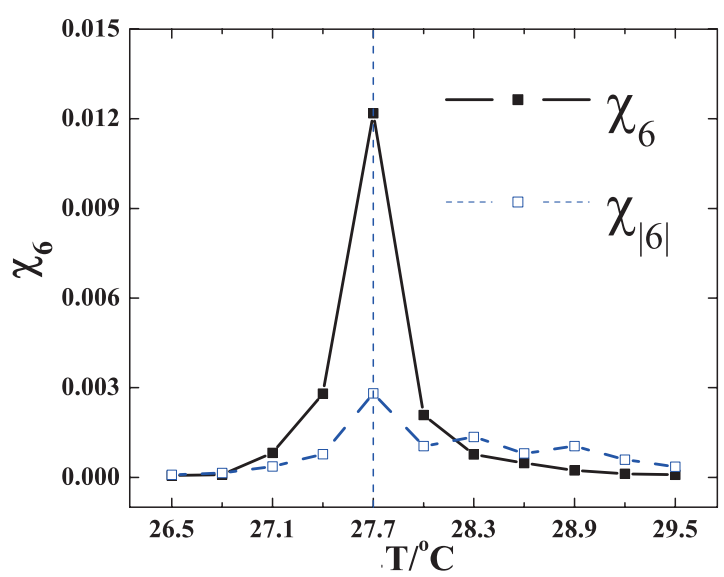

FIG. 8. Solid squares: The orientational susceptibility $\chi_{6}$, i.e., the fluctuation of the order parameter $\psi_{6}$. Open squares: the fluctuation of $\psi_{|6|}$.

distribution ${ }^{10}$ forms at about $28.4{ }^{\circ} \mathrm{C}$; the area fraction at this temperature is about $6 \%$ lower than that at $27.7^{\circ} \mathrm{C}$. This observation is thus consistent with previous work about hard disks; ${ }^{10,12}$ in previous work the bimodal distribution at $\pi n \sigma^{2} / 4=0.65$ also appeared at area fractions $6 \%$ lower than the hard-disk freezing point of 0.69 . At the hard-disk freezing point, the second peak in the shape factor distribution almost disappears in Refs. 10 and 12, thereby corresponding quite well to the observed shape at $27.7^{\circ} \mathrm{C}$ in Fig. 7. Hence, observations in our polycrystalline system agree reasonably well with simulations.

\section{E. Orientational susceptibility at the freezing point}

We observed two other interesting behaviors at the freezing point which could potentially serve as freezing criteria, if they are found to hold universally for different particle interactions. The first observation is that the freezing transition occurs at the apparent divergence point of the orientational order-parameter susceptibility. The orientational susceptibility is defined as

$$
\chi_{6}=A\left(\left\langle\left|\psi_{6}^{2}\right|\right\rangle-\left\langle\left|\psi_{6}\right|\right\rangle^{2}\right) .
$$

Here $\psi_{6}=\left\langle\psi_{6 j}\right\rangle=\left(\sum_{j=1}^{N} \psi_{6 j}\right) / N$ is the total order parameter averaged over all $N$ particles in the area $A . \psi_{6}$ is 0 in liquid and is close to unity in a single crystal. The susceptibility divergence has been used previously to identify 2D melting phase transitions in simulations ${ }^{42,43}$ and experiments. ${ }^{25}$ In previous work, the susceptibility method (assuming sufficient signalto-noise) has been shown to be superior in many respects to measurements of space- and time-correlation functions for assignment of transition points, primarily because the former method has little finite-size/time ambiguity. ${ }^{25}$ Until now the susceptibility technique has only been applied to singlecrystal melting and freezing.

For polycrystalline solids, the complex numbers $\psi_{6 j}$ of particles from different domains cancel in the averaging process, so that $\psi_{6}$ and $\chi_{6}$ approach 0 in the infinite sample-size limit. Figure 2(b) shows that $\psi_{6}$ of the polycrystalline solid in the field of view is only about 0.15 , much less than 1 . However $\chi_{6}$ in Fig. 8 still exhibits a clear peak at the $27.7{ }^{\circ} \mathrm{C}$ freezing point. If we use $\left|\psi_{6 j}\right|$ instead of $\psi_{6 j}$ for each
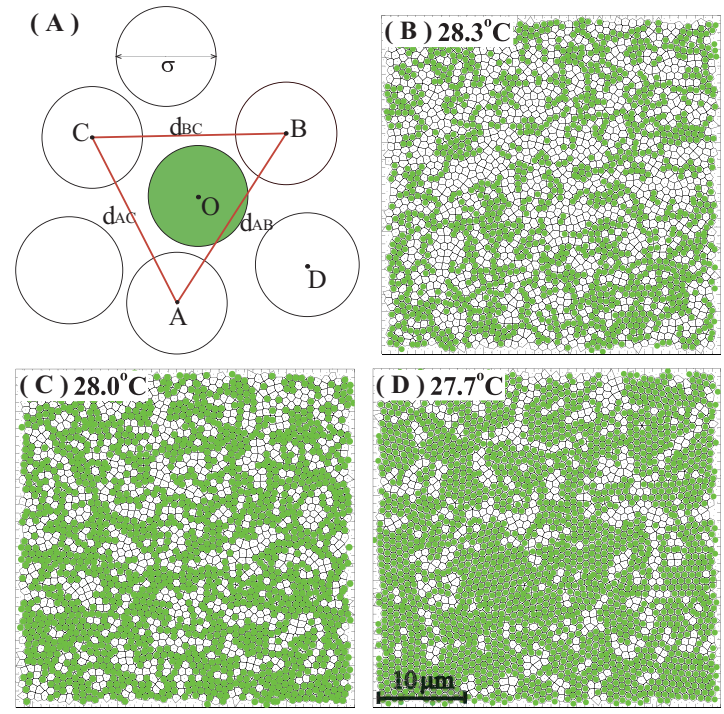

FIG. 9. (a) The central disk is caged by the three neighbors if $O$ is in $\triangle A B C$ and $2 \sigma>\max \left(d_{A B}, d_{B C}, d_{C A}\right) . \sigma$ is the particle diameter. (b) The Voronoi diagram of a subarea at $28.3^{\circ} \mathrm{C}$ below the percolation point. Caged particles are colored in green. (c) Voronoi cells of caged particles percolate through the field of view at $28.0{ }^{\circ} \mathrm{C}$. (d) Above the finite-area percolation point. In fact, $27.7^{\circ} \mathrm{C}$ is the true percolation point after the extrapolation to the infinite area [see inset of Fig. 10(c)].

particle $j$, then $\psi_{|6|}=\left\langle\left|\psi_{6 j}\right|\right\rangle$ will more readily distinguish polycrystalline solids from liquids. In a polycrystalline solid, most particles have six ordered neighbors and are not located at grain boundaries, and thus $\psi_{|6|}$ is close to 1 [see Fig. 2(b)]. The corresponding $\chi_{|6|}=\left\langle\left|\psi_{|6|}\right|^{2}\right\rangle-\left\langle\left|\psi_{\mid 6}\right|\right\rangle^{2}$ also peaks at $27.7^{\circ} \mathrm{C}$, see Fig. 8. Note, the peak temperature remains unchanged for $\chi_{6}$ and $\chi_{|6|}$ measured from different sized subareas. Hence, the susceptibility parameters can be used as freezing criteria. (As an aside, it should be noted that we cannot apply the same approach for the translational susceptibility, since the primary reciprocal lattice vector of a polycrystalline solid is not unique.)

\section{F. Caged particle percolation at the freezing point}

The last interesting feature we have discovered at the freezing point is a percolation transition of caged particles. Caging is a key concept in the cell model for the freezing transition. ${ }^{44}$ The cell model is based on free volume calculations, and it has successfully predicted the free energies, ${ }^{45}$ the dynamical Lindemann parameter, and the equation of state $^{46}$ at freezing. The definition of caging has evolved from six-neighbor caging in the conventional cell model ${ }^{47}$ to fourneighbor caging in the correlated cell model ${ }^{46}$ to the recent three-alternating-neighbor caging ${ }^{48}$ defined in Fig. 9(a).

Briefly, a central particle is said to be caged if any three alternating neighbors out of the six nearest neighbors satisfies the following two conditions: (1) the center of the central particle lies inside the triangle of the three neighbors; (2) the center-to-center distances of the three neighbors, $d_{A B}, d_{B C}$, $d_{C A}$, are smaller than two particle diameters (i.e., the central particle cannot squeeze out of the triangle). We further generalize this caging definition to any three neighbors out of an arbitrary number of neighbors so that a small fraction of particles without six neighbors can also be accounted for. For 

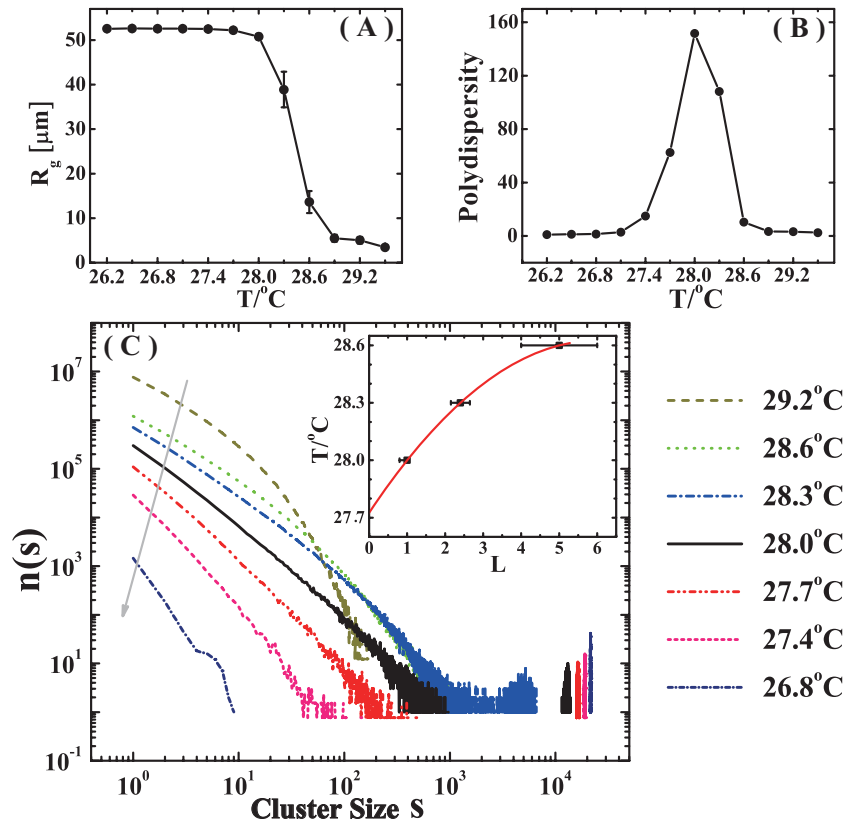

$29.2^{\circ} \mathrm{C}$

$28.6^{\circ} \mathrm{C}$

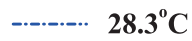

$-28.0^{\circ} \mathrm{C}$

$-\cdots \cdots-27.7^{\circ} \mathrm{C}$

$27.4^{\circ} \mathrm{C}$

$26.8^{\circ} \mathrm{C}$

FIG. 10. (a) The radius of gyration of the largest clusters averaged over all frames. Error bars are the standard deviations of the largest-cluster radii in different frames. (b) The polydispersity of the size of all clusters in all frames. (c) The histogram of cluster size distributions at different temperatures. The arrow indicates decreasing temperature. Inset: Finite-area percolation temperatures at different sub-boxes with area $A_{0} / L^{2}$, where $A_{0}$ is the area of the whole field of view and $1 / L$ is the contraction factor of the box. The real percolation temperature is extrapolated to the infinite-sized $(L=0)$ limit by a quadratic fit.

example, if a particle has five nearest neighbors, choosing three out of five results in 10 possible triangles. If any one of the ten triangles can cage the central particle, then we say the central particle is caged and color its Voronoi polygon green [see Fig. 9(b)]. The above construction is mathematically precise without ambiguity. Note, this caging definition is based purely on local static structure, i.e., rather than caging defined by the dynamic mean square displacement. ${ }^{49}$

The number of caged particles was found to increase with area fraction, and the Voronoi cells of the caged particles were found to percolate through the finite field of view at $28.0^{\circ} \mathrm{C}$. After extrapolation to infinite area (see below for details of the extrapolation procedures), the percolation point shifts to $27.7^{\circ} \mathrm{C}$. The percolation point in the finite field of view was obtained by all of the following three methods as shown in Figs. 10(a)-10(c).

The first method measured the radius of gyration of the largest cluster in each frame. The radius of gyration $R_{g}$ is defined as $R_{g}^{2}=\sum_{n=1}^{s}\left(r_{n}-r_{0}\right)^{2} / s$ where $r_{n}$ is the position of the $n$th particle in the cluster with $s$ particles; $r_{0}$ is the position of the center of mass. Figure 10(a) shows the mean values of $R_{g}$ averaged over all video frames and the error bars represent standard deviations. At temperatures above $28.0^{\circ} \mathrm{C}$, the mean largest-cluster size becomes less than the size of the field of view, suggesting that the percolation temperature is at $28.0{ }^{\circ} \mathrm{C}$.

The second method is based on polydispersity of clustersize. Results are indicated in Fig. 10(b) which shows that the cluster-size polydispersity peaks at the percolation point. The cluster-size polydispersity is defined as $\left\langle s^{2}\right\rangle /\langle s\rangle^{2}$ where $\langle s\rangle$ is the mean cluster size averaged over all clusters in all frames. This polydispersity reflects the size fluctuation of all clusters, not just the largest clusters in each frame. In fact the interpolated peak in Fig. 10(b) is between 28.0 and $28.3{ }^{\circ} \mathrm{C}$. We found that the interpolated peak is reflective of percolation through the finite sample-size, and that this point shifted continuously to higher temperature when the size of the sample subarea was decreased.

A third method derives the percolation temperature from the distribution, $n(s)$, of cluster size $s$, see Fig. 10(c). In the percolation theory, ${ }^{50} n(s) \propto s^{-\tau} e^{-c s}$ where the constant $\tau$ is the Fisher exponent. When approaching the percolation transition, $c \rightarrow 0$ and $n(s) \propto s^{-\tau} .^{50}$ The data in Fig. 10(c) suggest that the percolation point is about $28.0^{\circ} \mathrm{C}$, wherein $n(s)$ exhibits power-law decay. This observation is further confirmed by the emergence of a small peak of large clusters at $28.0^{\circ} \mathrm{C}$, see the $s>10^{4}$ regime in Fig. 10(c). Such a peak of large clusters is a direct evidence of percolation in a finite box. Empirically, we have found that this third method is very accurate when the field of view contains more than 5000 particles and, therefore, when the statistics of $n(s)$ are good. Since smaller areas percolate more easily, we repeated the above three measurements in different subareas of the sample and then extrapolated to the infinite-size limit. In this way we obtained the "truer" percolation point of $27.7^{\circ} \mathrm{C}$, see the inset of Fig. 10(c).

The percolation of caged particles qualitatively agrees with the notion of a solidification transition at the freezing point, even in polycrystalline samples. A caged particle cannot move much unless one of its three confinement neighbors moves, i.e., the caged particle must move in a correlated manner with its neighbor. At the percolation point, it becomes very difficult to collectively move many caged particles. Therefore, the system freezes. This general idea shares similarities with the ideas of dynamic heterogeneity in glass formation.

Lastly, we explored the potential fractal properties of the percolation clusters. A cluster is a fractal when its radial distribution of mass from the center of the cluster satisfies the power law $N=\left(r / R_{g}\right)^{d}$, where $d$ is the Hausdorff fractal dimension; the "mass" $N$ is the number of particles inside the circular box with radius $r$ from the center of mass of the cluster. In order to identify whether the largest cluster of caged particles in each frame is a fractal or not, we measured 10 masses in 10 circular boxes with radii $r=R_{g} / 10,2 R_{g} / 10, \cdots, R_{g}$. We repeated these measurements on the 3500 largest clusters in 3500 frames at each temperature. Therefore, each plot in Fig. 11 contains $3500 \times 10$ data points.

At 27.7 and $28.0{ }^{\circ} \mathrm{C}$, all the 3500 clusters have similar $R_{g}$ 's and similar mass distributions so that the $3500 \times 10$ data points collapse onto the 10 discrete points, see Figs. 11(b) and 11(c). The power-law mass distributions in Figs. 11(b) and $11(\mathrm{c})$ indicate that the percolation clusters at 27.7 and $28.0^{\circ} \mathrm{C}$ are good fractals. From the slope of Fig. 11(c), we obtained the fractal dimension $d=1.97 \pm 0.016$ at the percolation point, close to the $d=1.91 \pm 0.04$ result for the continuum percolation of $2 \mathrm{D}$ random hard disks. ${ }^{51}$ At $28.3^{\circ} \mathrm{C}$, the $3500 \times 10$ data points in Fig. 11(a) are not fractal; the 

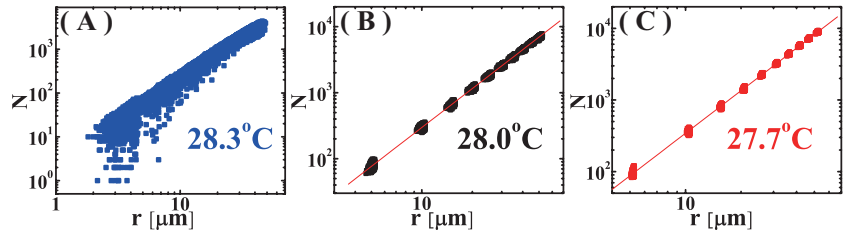

FIG. 11. The mass distributions of the 3500 largest clusters in 3500 frames at (a) $28.3{ }^{\circ} \mathrm{C}$, (b) $28.0^{\circ} \mathrm{C}$, and (c) $27.7^{\circ} \mathrm{C}$. For each largest cluster in a frame, the mass distribution is measured from the masses inside the 10 circular areas with radii $r=R_{g} / 10,2 R_{g} / 10, \ldots, R_{g}$, where $R_{g}$ is the cluster radius of gyration. Each plot contains $3500 \times 10$ data points measured from 3500 frames. Clusters at $28.3{ }^{\circ} \mathrm{C}$ are not fractal because each set of 10 data points in (a) does not follow a power law $N=\left(r / R_{g}\right)^{d}$ where $d$ is the Hausdorff fractal dimensions. Clusters at 28.0 and $27.7^{\circ} \mathrm{C}$ are fractals because each set of 10 data points in (b) and (c) follows a power law.

$3500 R_{g}$ 's fluctuate too strongly. In percolation theory, a random percolation cluster is a fractal. ${ }^{50}$ However the caged particles are not totally random, because they have underlying polycrystalline structures. Interestingly Fig. 11 shows that the percolation clusters of caged particles are still good fractals.

Similar fractal structures of different types of percolation clusters were reported in 2D glass transitions and such fractal structures were used to explain many hallmark properties of glassy dynamics. ${ }^{52,53}$ We speculate that it will be interesting to explore whether the fractal structures of caged particles are related to the dynamics near the polycrystalline solid (or single-crystal) freezing point. Structure heterogeneity in 2D single-crystal melting has been discussed in terms of a disorder parameter and density fluctuations,${ }^{54}$ but, to our knowledge, percolation and fractal structure have not been reported. For example, if we apply the caging concept to 2D melting, then the percolation transition of caged particles might be found to play a role during the melting.

\section{CONCLUSIONS}

We measured the polycrystalline freezing of microgel colloidal monolayers. The freezing appeared to be a firstorder process, without hexatic phase. This work provides the first experimental tests of four simulation-derived empirical criteria for freezing of thermal 2D liquids into 2D polycrystalline solids. Although the four criteria were originally proposed from simulations of single-crystal melting, the experiments demonstrate that these criteria also hold for the freezing into polycrystalline solids. Thus the results are useful, in part, because most experimental systems directly freeze into polycrystalline solids rather than single crystals. The observation also provides another point of view for study of the glass transition, e.g., by analyzing polycrystalline freezing of different domain sizes and then extrapolating to the small-domain limit. The HV and the LPS freezing criteria have been experimentally tested in $2 \mathrm{D}$ for the first time, and they appeared to be more accurate than criteria 3 and 4. The HV criterion depends on the global structure, while the criteria 3 and 4 only depend on local structures in real space. Since freezing is a collective arrangement of particles, the 2D HV freezing rule associated with global structure might have been expected to be the more accurate criterion, as observed in the experiment. We observed peaking of the susceptibilities, $\chi_{6}$ and $\chi_{|6|}$, at the same freezing point, even though the orientational order parameter is small after averaging over many domains. Another apparent signature at the freezing transition is the percolation of caged particles. The fractal structures of percolation clusters reflect structural and dynamical heterogeneities in 2D freezing. ${ }^{54,55}$ Furthermore, the caging definition is readily generalized to $3 \mathrm{D}$ where one sphere is caged by a tetrahedron of four neighbors. In the future, it may be interesting to study the prevalence of caged-particle percolation in $3 \mathrm{D}$ freezing and at the glass transition.

\section{ACKNOWLEDGMENTS}

We thank the helpful discussion with Yong Chen. This work was supported by Hong Kong SAR RGC Grant No. 601208, by the William Mong Institute of Nano Science and Technology at HKUST (Y.H.), and by the National Science Foundation (A.G.Y.) through Grant No. DMR-080488 and the PENN MRSEC Grant No. DMR-0520020.

${ }^{1}$ H. Löwen, Phys. Rep. 237, 249 (1994).

${ }^{2}$ U. Gasser, J. Phys.: Condens. Matter 21, 203101 (2009).

${ }^{3}$ F. A. Lindemann, Z. Phys. 11, 609 (1910).

${ }^{4}$ V. M. Bedanov, G. V. Gadiyak, and Y. E. Lozovik, Phys. Lett. A 109, 289 (1985).

${ }^{5}$ J. Q. Broughton, G. H. Gilmer, and J. D. Weeks, Phys. Rev. B 25, 4651 (1982).

${ }^{6}$ H. Löwen, T. Palberg, and R. Simon, Phys. Rev. Lett. 70, 1557 (1993). ${ }^{7}$ H. Löwen, Phys. Rev. E 53, R29 (1996).

${ }^{8}$ S. Ranganathan and K. N. Pathak, Phys. Rev. A 45, 5789 (1992).

${ }^{9}$ T. M. Truskett, S. Torquato, S. Sastry, P. G. Debenedetti, and F. H. Stillinger, Phys. Rev. E 58, 3083 (1998).

${ }^{10}$ F. Moučka and I. Nezbeda, Phys. Rev. Lett. 94, 040601 (2005).

${ }^{11}$ G. P. Hoffmann and H. Löwen, J. Phys.: Condens. Matter 13, 9197 (2001).

${ }^{12}$ P. M. Reis, R. A. Ingale, and M. D. Shattuck, Phys. Rev. Lett. 96, 258001 (2006).

${ }^{13}$ J. M. Kosterlitz and D. J. Tholuless, J. Phys. C 6, 1181 (1973).

${ }^{14}$ D. R. Nelson and B. I. Halperin, Phys. Rev. B 19, 2457 (1979).

${ }^{15}$ A. P. Young, Phys. Rev. B 19, 1855 (1979).

${ }^{16}$ D. R. Nelson, Defects and Geometry in Condensed Matter Physics (Cambridge University Press, Cambridge, 2002).

${ }^{17}$ M. A. Glaser and N. A. Clark, Adv. Chem. Phys. 83, 543 (1993).

${ }^{18}$ S. T. Chui, Phys. Rev. Lett. 48, 933 (1982).

${ }^{19}$ S. T. Chui, Phys. Rev. B 28, 178 (1983).

${ }^{20}$ K. J. Strandburg, Rev. Mod. Phys. 60, 161 (1988).

${ }^{21}$ H. König, R. Hund, K. Zahn, and G. Maret, Eur. Phys. J. B 18, 287 (2005).

${ }^{22}$ C. S. O'Hern, S. A. Langer, A. J. Liu, and S. R. Nagel, Phys. Rev. Lett. 86, 111 (2001)

${ }^{23}$ Z. Zhang, N. Xu, D. T. N. Chen, P. Yunker, A. M. Alsayed, K. B. Aptowicz, P. Habdas, A. J. Liu, S. R. Nagel, and A. G. Yodh, Nature (London) 459, 230 (2009).

${ }^{24}$ K. Zahn, R. Lenke, and G. Maret, Phys. Rev. Lett. 82, 2721 (1999).

${ }^{25}$ Y. Han, N. Y. Ha, A. M. Alsayed, and A. G. Yodh, Phys. Rev. E 77, 041406 (2008).

${ }^{26}$ C. A. Murray and D. H. Van Winkle, Phys. Rev. Lett. 58, 1200 (1987).

${ }^{27}$ A. H. Marcus and S. A. Rice, Phys. Rev. Lett. 77, 2577 (1996).

${ }^{28}$ D. G. Grier and C. A. Murray, J. Chem. Phys. 100, 9088 (1994).

${ }^{29}$ U. Gasser, E. R. Weeks, A. Schofield, P. N. Pusey, and D. A. Weitz, Science 292, 258 (2001).

${ }^{30}$ J. P. Hoogenboom, P. Vergeer, and A. van Blaaderen, J. Chem. Phys. 119, 3371 (2003).

${ }^{31}$ K. Q. Zhang and X. Y. Liu, Nature (London) 429, 739 (2004).

${ }^{32}$ J. R. Savage and A. D. Dinsmore, Phys. Rev. Lett. 102, 198302 (2009).

${ }^{33}$ P. Dillmann, G. Maret, and P. Keim, J. Phys.: Condens. Matter 20, 404216 (2008).

${ }^{34}$ C. A. Knapek, D. Samsonov, S. Zhdanov, U. Konopka, and G. E. Morfill, Phys. Rev. Lett. 98, 015004 (2007). 
${ }^{35}$ Y. Feng, J. Goree, and B. Liu, Phys. Rev. Lett. 100, 205007 (2008).

${ }^{36}$ J. C. Crocker and D. G. Grier, J. Colloid Interface Sci. 179, 298 (1996).

${ }^{37}$ A. Z. Patashinski, A. C. Mitus, and M. A. Ratner, Phys. Rep. 288, 409 (1997).

${ }^{38}$ J. M. Caillol, D. Levesque, J. J. Weis, and J. P. Hansen, J. Stat. Phys. 28, 325 (1982).

${ }^{39}$ J.-P. Hansen and L. Verlet, Phys. Rev. 184, 151 (1969).

${ }^{40}$ R. C. Gann, S. Chakravarty, and G. V. Chester, Phys. Rev. B 20, 326 (1979).

${ }^{41}$ R. Pesché, M. Kollmann, and G. Nägele, J. Chem. Phys. 114, 8701 (2001).

${ }^{42}$ H. Weber, D. Marx, and K. Binder, Phys. Rev. B 51, 14636 (1995).

${ }^{43}$ R. Zangi and S. A. Rice, Phys. Rev. E 58, 7529 (1998).

${ }^{44}$ J. A. Barker, Lattice Theory of the Liquid State (Pergamon, New York, 1963).

${ }^{45}$ W. G. Hoover and F. H. Ree, J. Chem. Phys. 49, 3609 (1968).

${ }^{46}$ B. J. Alder, W. G. Hoover, and T. E. Wainwright, Phys. Rev. Lett. 11,
241 (1963).

${ }^{47}$ Y. Wang, T. Ree, T. S. Ree, and H. Eyring, J. Chem. Phys. 42, 1926 (1965).

${ }^{48}$ A. Huerta, D. Henderson, and A. Trokhymchuk, Phys. Rev. E 74, 061106 (2006).

${ }^{49}$ E. R. Weeks, J. C. Crocker, A. C. Levitt, A. Schofield, and D. A. Weitz, Science 287, 627 (2000).

${ }^{50}$ D. Stauffer and A. Aharony, Introduction to Percolation Theory (Taylor \& Francis, London, 1994).

${ }^{51}$ E. T. Gawlinski and H. E. Stanley, J. Phys. A 14, L291 (1981).

${ }^{52}$ G. Lois, J. Blawzdziewicz, and C. S. O’Hern, Phys. Rev. Lett. 100, 028001 (2008).

${ }^{53}$ G. Lois, J. Blawzdziewicz, and C. S. O'Hern, Phys. Rev. Lett. 102, 015702 (2009).

${ }^{54}$ H. Shiba, A. Onuki, and T. Araki, Europhys. Lett. 86, 66004 (2009).

${ }^{55}$ B. Cui, B. Lin, and S. A. Rice, J. Chem. Phys. 114, 9142 (2001). 